\title{
A survey on the current status and future perspective of informed consent management in the MIRACUM consortium of the German Medical Informatics Initiative
}

Christopher Hampf ${ }^{1 * \dagger}$ (D) Martin Bialke ${ }^{1 \dagger}$, Lars Geidel ${ }^{2}$, Albert Vass $^{1}$, Thomas Bahls $^{1}$, Romina Blasini ${ }^{3}$, Arne Blumentritt ${ }^{2}$, Martin Boeker ${ }^{4}$, Christian Bruns ${ }^{5}$, Burkhard Jandrig ${ }^{6}$, Maximilian Fünfgeld ${ }^{7}$, Philipp Heinrich ${ }^{8}$, Torsten Leddig ${ }^{1}$, Achim Michel-Backofen ${ }^{9}$, Anna Pirkl ${ }^{10}$, Michael Rautenberg ${ }^{11}$, Fabian Simons ${ }^{12}$, Dana Stahl ${ }^{2}$, Hans-Ulrich Prokosch ${ }^{13}$ and Wolfgang Hoffmann ${ }^{1}$

\begin{abstract}
Background: The consent management is an essential component for supporting the implementation of consents and withdrawals and thus, the realisation of patient's rights. In MIRACUM, one of the four consortia of the Medical Informatics Initiative (MII), ten university hospitals intend to integrate the generic Informed Consent Service ${ }^{\circledast}$ (gICS) in their Data Integration Center (DIC). To provide a tool that supports the local workflows of the MIRACUM sites, the glCS should be improved.

Methods: We used three standardised questionnaires with 46 questions to elicit requirements from the ten sites. Each site answered the questions from the current and the desired future perspective. This made it possible to understand the individual processes at each site and it was possible to identify features and improvements that were generally necessary.

Results: The results of the survey were classified according to their impact on the gICS. Feature requests of new functionalities, improvements of already implemented functionalities and conceptual support for implementing processes were identified. This is the basis for an improved glCS release to support the ten sites' individual consent management processes.

Conclusions: A release plan for the feature requests and improvements was coordinated with all sites. All sites have confirmed that the implementation of these features and enhancements will support their software-based consent management processes.
\end{abstract}

Keywords: Consent management, Informed consent, General data protection regulation, GDPR, Medical informatics initiative, MIRACUM

\footnotetext{
* Correspondence: christopher.hampf@uni-greifswald.de

${ }^{\dagger}$ Christopher Hampf and Martin Bialke contributed equally to this work.

'Institute for Community Medicine, University Medicine Greifswald,

Ellernholzstr. 1-2, 17475 Greifswald, Germany

Full list of author information is available at the end of the article
}

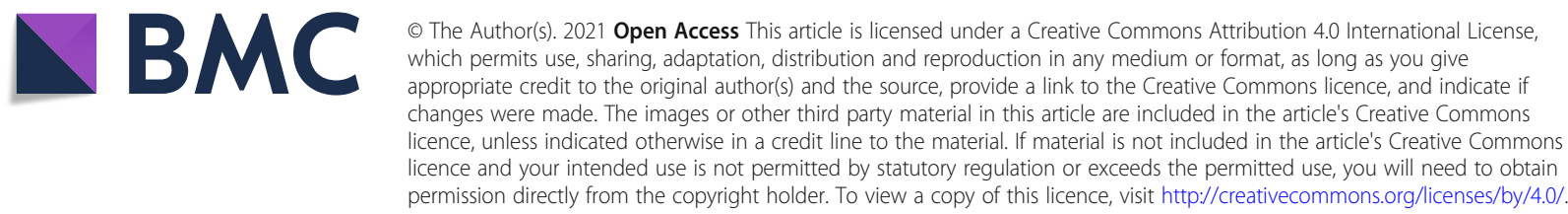




\section{Background}

The German Federal Ministry of Education and Research (BMBF) funded in its Medical Informatics Initiative (MII) the four consortia MIRACUM [1], HiGHmed [2], SMITH [3] and DIFUTURE [4] to improve medical research and patient care with innovative data architectures and software solutions. Each consortium comprises an association of academic and hospital partners as well as industrial partners. In order to address MII-wide problem solutions across all sites, working groups have been established by the Steering Committee of the MII for various tasks, e.g. the working group consent (for the coordination of a uniform and modular consent document) and the task force consent implementation (for the harmonized and interoperable implementation of electronic consents) $[5,6]$. The corresponding solutions will be tested and implemented by all consortia and related partner sites. MIRACUM includes ten University hospitals and one industrial partner. In each site, a Data Integration Center (DIC) will be established. These DIC combine open-source software solutions to enable data sharing for multi-site medical research. This requires an EU GDPR-conform processing of a patient's (or a study participant) identifying and medical data to ensure the patient's rights. If there is no legal basis for a research purpose, an informed consent (IC) is required (GDPR Art. 6 (1) lit. a). In the case of multi-site research, only the data of patients or participants who have consented to this specific research purpose may be used. Moreover, a patient needs to be informed about the consequences of consent. This includes that the concerned person has the opportunity to ask questions for a better understanding of the data processing steps. Patients and participants can withdraw a given consent at any time and without stating a reason (GDPR Art. 7 (3)) [7]. Hence, applying a reliable dynamic consent management at each MII site is recommended, which ensures that consents are documented, managed, and stored in a timely and traceable manner. The realisation of a withdrawal is more of an organisational issue, but requires the support of technical tools. Several hundred partial or complete withdrawals per site and year cannot be processed manually. In addition, partial withdrawals have to be supported. In a case, that a patient does not want to be contacted to be informed about upcoming studies or scientific results. Partial withdrawals allow to comply to this patient will while still being able to use the collected data scientifically.

The consent management is an essential component in supporting the realisation of the patient's rights. At present consents or withdrawals are still paper-based, which makes automated processing difficult [8]. Consents are also often customised for a specific study, which limits the comparability [8]. A digitalised consent management enables a research institution to organise consents centrally and allows easy searches for patients with a specific consent state [9]. For a uniform representation and comparability, the MII working group for consent elaborated a uniform broad consent that will be used over all sites in the four consortia [6].

In addition to a DIC, each site has to establish a local Trusted Third Party (TTP). The TTP is responsible for managing identities, pseudonyms and consents for research projects [10]. Bialke et al. descripted the typical TTP infrastructure, technical as well as organisational aspects and involved stakeholders in terms of research data management [10]. Each MIRACUM site plans to implement a TTP by the end of 2020. Established software solutions should support the tasks of a TTP. Within MIRACUM, various available open-source tools are orchestrated in a collection called Medical Informatics ReusAble eCosystem of Open source Linkable and Interoperable software tools (MIRACOLIX) [1, 11]. The generic Informed Consent Service $\left(\mathrm{gICS}^{\oplus}\right)$ [8] is one part of this collection. gICS follows the principles of "privacy by design" $[10,12]$ and supports the digital- and paperbased management of consents. It was developed by the University Medicine Greifswald within the GANI_MED [13] project and published in the MOSAIC [14] project, which was funded by the German Research Foundation (HO 1937/2-1). It is presently used in a large number of research projects and further developed following the new requirements. It handles modular consents that are composed of re-usable modules and policies [9, 10]. The gICS provides the actual status of each consent policy for every patient at any given point in time. The status is updated if the patient changed parts of their consent forms or completely withdraws from their participation in a study [8]. The digital collection of consents is supported by the opportunity to sign electronically and store relevant information to reproduce signatures. Moreover, paper-based documentation is supported: consent templates can be printed, filled in by the patient in writing and then be scanned. The gICS recognizes the templates automatically and documents the contents with the respective study [9]. Rau et al. already compared gICS with several approaches for consent management in terms of advantages and disadvantages [8].

The University Medicine Greifswald successfully mastered legal, technical and organisational challenges in establishing a TTP and productively operated a TTP since 2014 [10]. Presently, the University Medicine Greifswald shares experiences with the MIRACUM partner sites to accelerate the TTP-implementation at each MIRACUM site. To support the implementation, the University Medicine Greifswald offers free workshops for all MIRACUM sites to train the handling of gICS and all processes in a TTP, including identity management, pseudonym management, record linkage (prevention of duplicate persons) and quality management. 
All ten sites of MIRACUM intend to use the gICS as consent management solution within their TTPs [15], this not only means the installation of a tool, but also the full integration of the gICS into the individual processes at the sites. It is not necessary that the staff collecting the consents be specially trained in the use of the gICS. Common use cases are described in the provided gICS manual [16]. Specific questions can be asked in the community dialog of the Trusted Third Party of the University Medicine Greifswald [17]. Each partner site has individual technical processes and infrastructures. In addition, to meet security requirements, various security measures must be implemented by the sites. Amongst others, this includes separation of networks (separate from clinical and research networks), implementation of authentication and authorization measures and common network restrictions (firewalls, IP filters, TLSv.1.2) [10]. In addition to the technical aspects, each TTP requires staff who may have site-specific responsibilities. TTP staff support workflows within the TTP, address the quality of consents, and resolve possible matches for record linkage. It is necessary to inspect the similarities and differences between the sites regarding local consent processes, IT-infrastructures and individual organizational frameworks [15]. This study aims to identify new requirements and future application areas for the consent management solution, translate these in feature requests, and implement these in upcoming gICS releases.

\section{Objectives}

The goal of this paper is to (I) analyse each single consent process of each site, (II) derive a set of new application scenarios and new requirements, which (III) are compared with existing functionalities of gICS and finally (IV) to plan the upcoming releases to provide the best possible support to the sites.

\section{Methods \\ Comparing current and future consent management processes}

In a two-step approach, we determined for all MIRA CUM partner sites (a) how consents were handled at an early stage of the MIRACUM project (with the perspective of January 2019) and (b) how consents shall be handled, supported by a TTP, in the near future (with the perspective of December 2020). The total of 46 questions were prepared in three questionnaires for this analysis, each aiming at a different topic, such as (1) information of the patient, (2) collection and documentation of consents and (3) documentation and realisation of withdrawals.

All questions were consolidated and jointly agreed with by all MIRACUM sites. The 46 questions were answered from both time perspectives (a) and (b) by all partner sites. Using questionnaires facilitated structured and categorized data capture of the requirements. Most questions were multiple-choice formats. Free text answers were avoided wherever possible. Two example multiple choice questions with their answer options are provided in Tables 1 and 2 .

All questionnaires were provided step by step in MIRACUM's internal web-based documentation software "Atlassian Confluence". Due to sites' individual characteristics the partners were encouraged to answer the questions from the perspective of the institution, the department or a certain research project. For the early perspective information(a), we surveyed the study staff and related researchers with regards to (among others):

- "how many consents are collected",

- "which kind of staff (physician, who performed patient information, trained/educated specialist, study staff or other) collects the consents", and

- "how much time is needed to collect the consent".

To gather the future perspective of consent management (b), all partners answered the same surveys supported by their DIC leaders and described their vision of the DIC at the end of 2020 based on the usage of gICS as the consent management solution. In total, gathering all MIRACUM site answers for the six surveys ( 3 topics $\times 2$ perspectives) took 6 months.

We ensured that all sites answered all questionnaires completely. For data analysis, we extracted all answers from the Confluence with the help of JavaScript and performed necessary quality assurance to guarantee the answers' syntactical comparability. We conducted a plausibility check, searched for inconsistencies and facilitated answering the given questions when necessary.

The quality approved survey data was analysed with $\mathrm{R}$ and $\mathrm{R}$ Studio, utilising various plugins like markdown, ggplot2, tibble, dplyr, etc. The developed script library was published for all MIRACUM sites afterwards to ensure reproducibility and traceability of all sites' analysis results.

Table 1 This question is included in the questionnaire for "patient information"

When and where is the patient informed?
- outside the institution / at home
- on admission to the institution
- during the information for an examination / treatment in the
institution
- after treatment in the institution
- others (please specify)


Table 2 This question is included in the questionnaire for "collection and documentation of consents"

\begin{tabular}{l} 
How much time does "giving consent" (obtaining, documenting/ \\
digitising) currently take on average per patient? \\
\hline - less than $5 \mathrm{~min}$ \\
- $5-10 \mathrm{~min}$ \\
-11-15 min \\
- $16-30 \mathrm{~min}$ \\
- $31-60 \mathrm{~min}$ \\
- more than $60 \mathrm{~min}$
\end{tabular}

\section{Considering future changes and similarities in consent management processes}

To identify significant changes and similarities in the assessed consent management processes of the MIRA CUM sites, we compared the current (a) and the future perspectives (b) based on the respective survey data.

The survey results let to comparison charts that show the current and further perspective and changes' tendencies.

We categorised the effect to gICS in respect of the changes between current (a) and further (b) perspective in:

- No change: the resulting feature is already implemented, or the topic is not relevant for the MIRACUM sites

- Minor change: already implemented features have to be extended

- Major change: new features have to be implemented

Furthermore, we could identify conceptual support. These do not require changes in the gICS, but require additional material, such as a template for specific consent.

As the last step, we evaluated how the most important goals of the MII partner sites within MIRACUM can be reached with the support of gICS. Thereby, it is important to distinguish between goals achievable by using an existing gICS release (Current version: 2.10.0), goals requiring new functionalities (feature request), several modifications (improvements) or simply conceptual support.

\section{Results}

The survey results highlight consent managementspecific topics where the MIRACUM sites expect no changes, minor changes or major changes of future consent-related work. For a better understanding, selected relevant results are shown briefly below.

1. Today, all sites check the correctness of the incoming consents. As a part of quality assurance, it is also relevant for the future perspective. Though
gICS (version 2.10.0) does not yet support quality assurance, new functionality to support consent quality assurance has to be implemented (classified as major change).

2. Regarding the documentation on patient information, currently in eight out of ten locations uses paper-based documentation. In the future, all ten sites plan to use digital documentation, with five of the sites planning to continue to use paper-based documentation additionally. This functionality requires an extension of the gICS and represents a major change (Fig. 1).

3. Figure 2 depicts a variety of information documented on withdrawal. This information will continue to be recorded in the future, in some cases even from significantly from more sites. The documentation of various information is not part of gICS and has to be implemented for this purpose. Because it is implemented by two different databases, the anonymisation and storing identifying data is not a conflict. In anonymisation, all identifying information in consent, pseudonym and identity management are deleted according to implementation of the withdrawal. To fulfil the obligation to provide evidence for the competent data protection officer, a separate database with all information is implemented. Only the employees of the TTP have access to the information. The process is classified as a major change.

4. Figure 3 shows that paper-based consent forms are currently being filled out, and a copy is given to a patient. This will continue to be the practice from eight sites in the future. Six locations would obtain informed consent in a digital process and hand out a digital consent print-out in the future. One location would like to send a print-out to the patient via postal service. Such kinds of large shift will be classified as a minor change as it requires improved features of the gICS. It is already possible to print digitally collected consents, but it is crucial to ensure that the print-out precisely represents the digital consent.

5. As shown in Fig. 4, today, almost all MIRACUM sites currently collect consents paper-based. In the future, additional technical possibilities apply, such as the fully electronic collection of consents or mixed approaches, including both electronic and paper-based elements. gICS already supports the digital management of consents and provides several features to support paper-based consent processing [9]. The survey reveals that processing of paper-based consents will still be relevant in the future and should therefore be optimally supported. This requires 


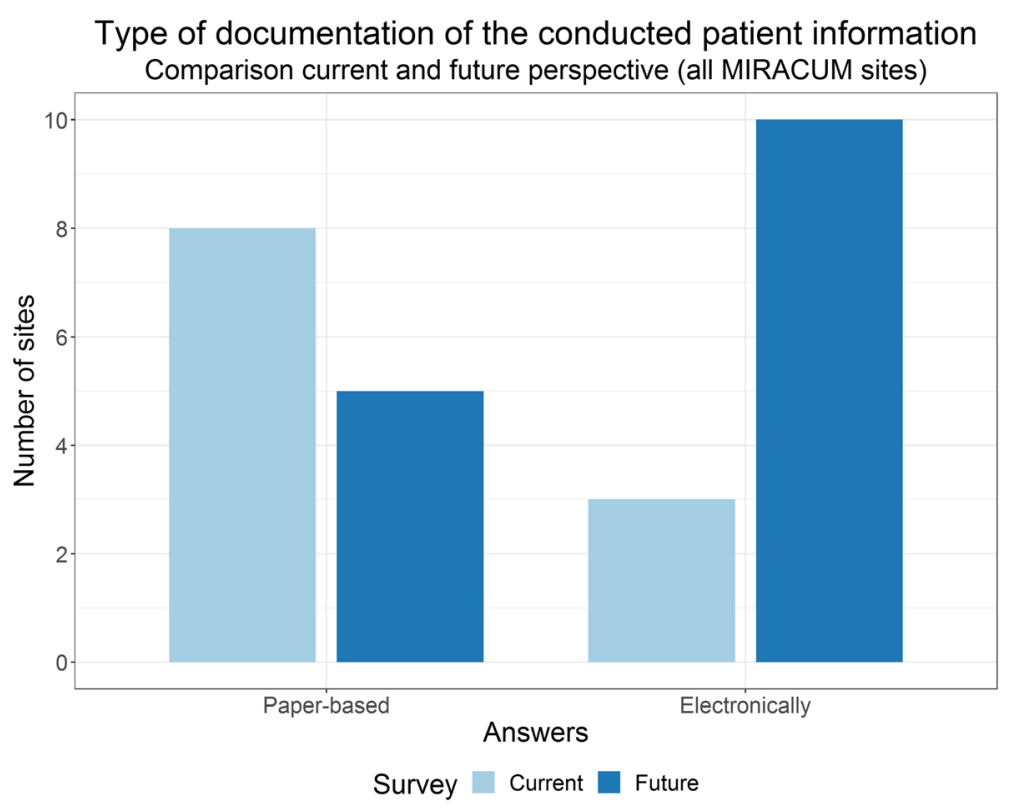

Fig. 1 Type of documentation of the conducted patient information. Frequency distribution of how the patient information is conducted currently and in future perspective

corresponding improvements and extensions of gICS and is classified as a minor change.

6. The realisation of a partial or complete withdrawal requires the notification of different systems, persons and units. As shown in Fig. 5, many of these are already being addressed to realise a withdrawal at the sites. In the future, the sites want to extend the number of connections for notifications. All sites will notify their trusted third party of the withdrawal for managing consents. As this becomes increasingly important for all sites, this change is classified as a minor change of the gICS.

7. From Fig. 6 it can be seen that all sites will record the documentation of withdrawals in a consent database in the future. Six sites indicate

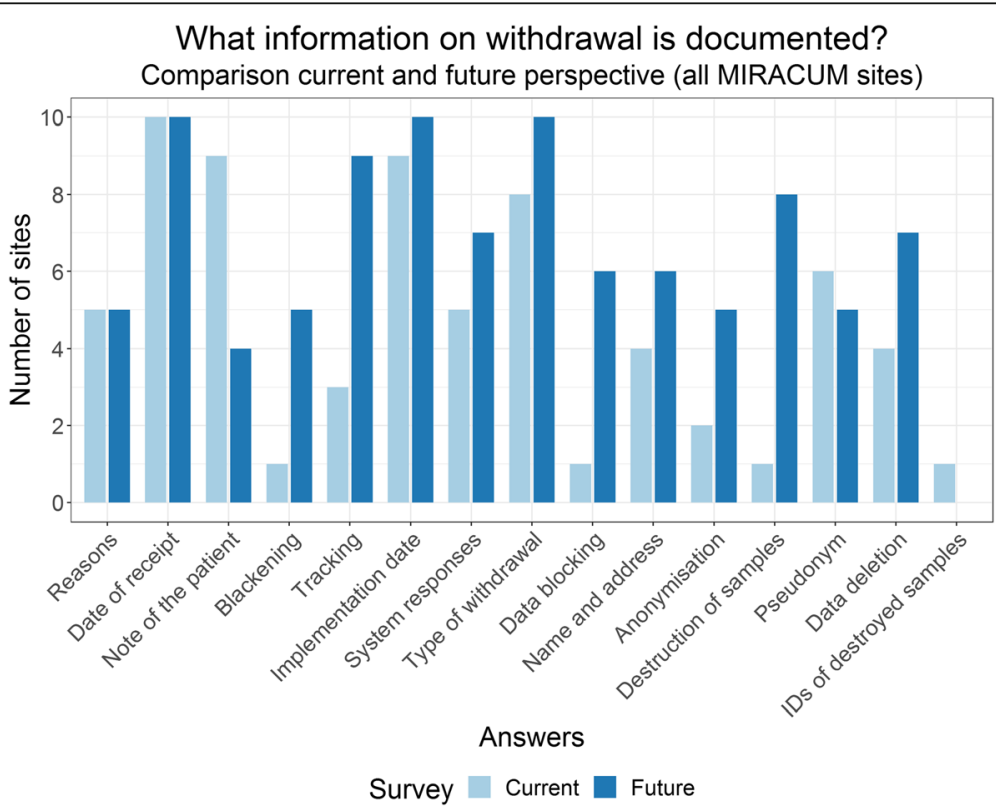

Fig. 2 What information on withdrawal is documented?. Frequency distribution which information for withdrawals are documented currently and in future perspective. (Blackening: making certain content unrecognizable) 


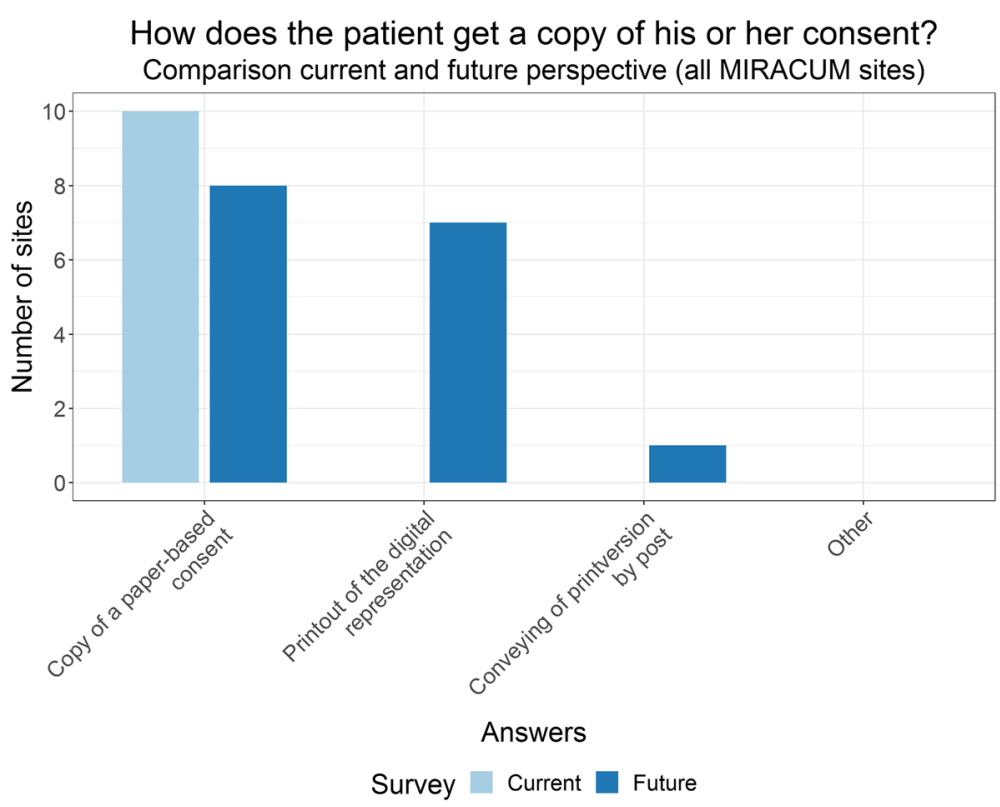

Fig. 3 How does the patient get a copy of his or her consent?. Frequency distribution of how patients get a copy of the filled out consent currently and in future perspective

that in future withdrawal information should additionally be recorded in the biobank or sample management. The management of withdrawals has to be supported accordingly in gICS. This requires a comprehensive extension of the gICS, which is classified as a major change.

8. Regarding the digitalisation of paper-based consents, four sites indicate that digitisation is not taking place on their site, while four sites indicate that they use different software solutions for digitisation. Two sites do not report their digitalisation status. For the future perspective, six sites plan to use various solutions to scan the consents. The consent management gICS, which is used across all sites, can already recognize the consent template and version. An extension by recognising the

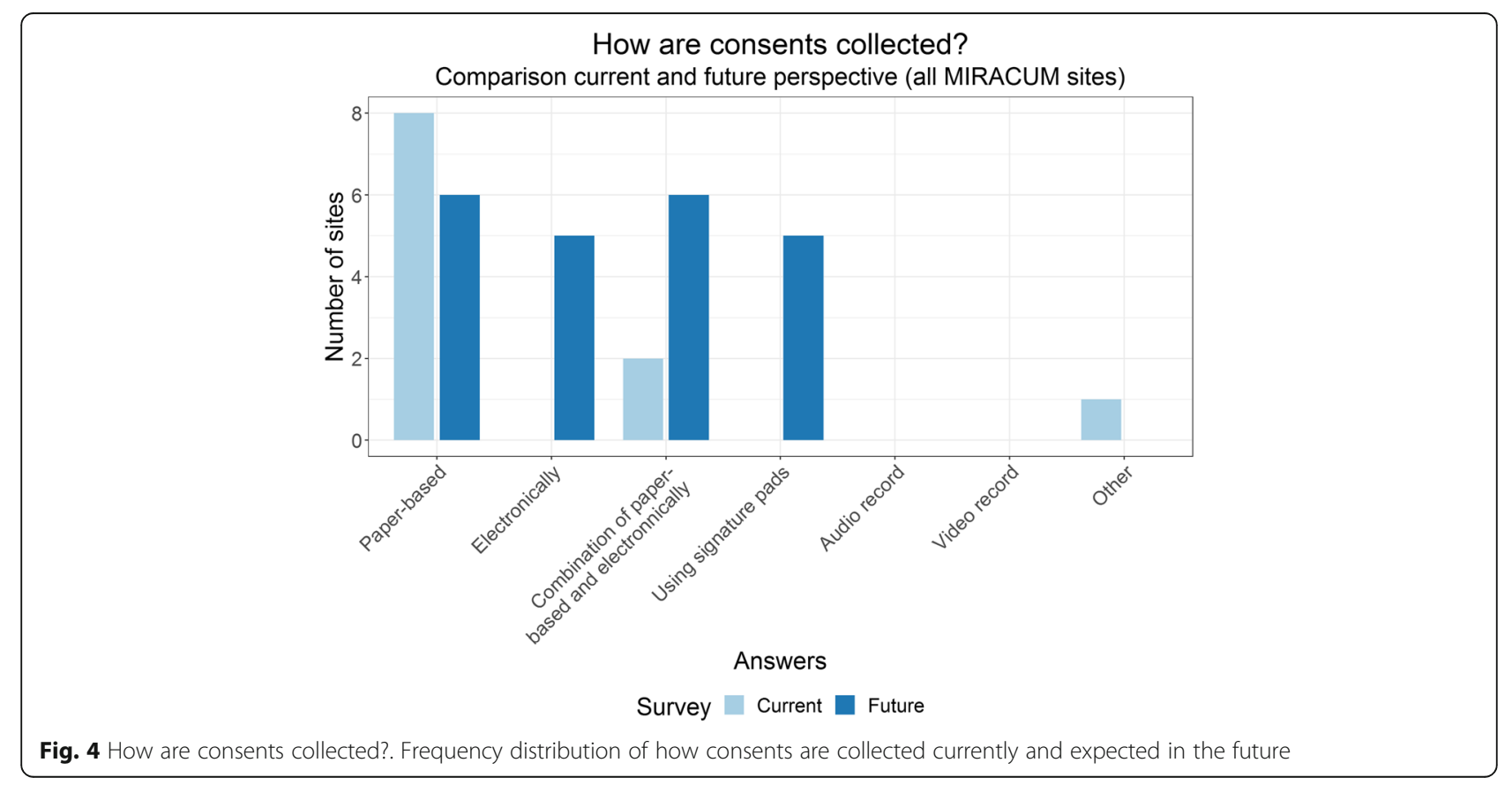


Which systems/persons/units have to be notified in case of a withdrawal?

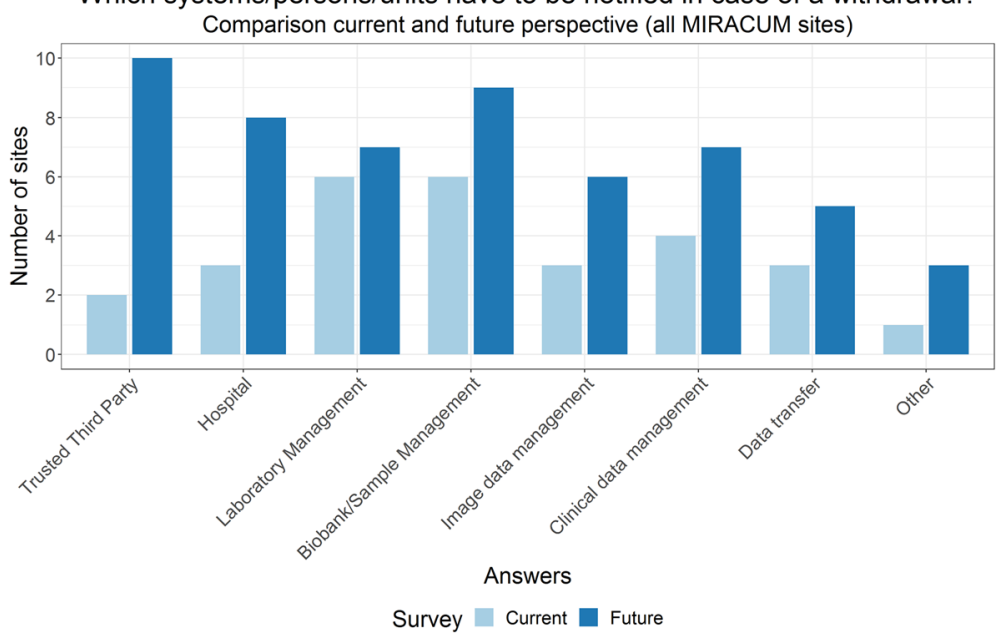

Fig. 5 Which systems/persons/units have to be notified in case of a withdrawal?. Frequency distribution of which systems, persons or units are informed about a partial or complete withdrawal currently and in future perspective

checked consent options would avoid further scan software and the manual entering of the consents in the gICS. This extension is classified as a minor change.

In total, we were able to identify three conceptual supports that do not require changes to the gICS, but do require content-related and procedural consulting/solution proposals from us.

9. In response to the question, "Who has access to the patient consents?" (see Table 3) various roles were mentioned. The implemented role and rights system of gICS supports different roles and privileges but has to be configured accordingly.

10. In response to the question, "What forms of withdrawal are used?" (see Table 4), seven sites indicated that they already implement partial withdrawals. Nine of the sites plans a future implementation. gICS support partial withdrawals, but a corresponding consent template has to be prepared. Concerning broad consent, a corresponding template is provided for gICS.

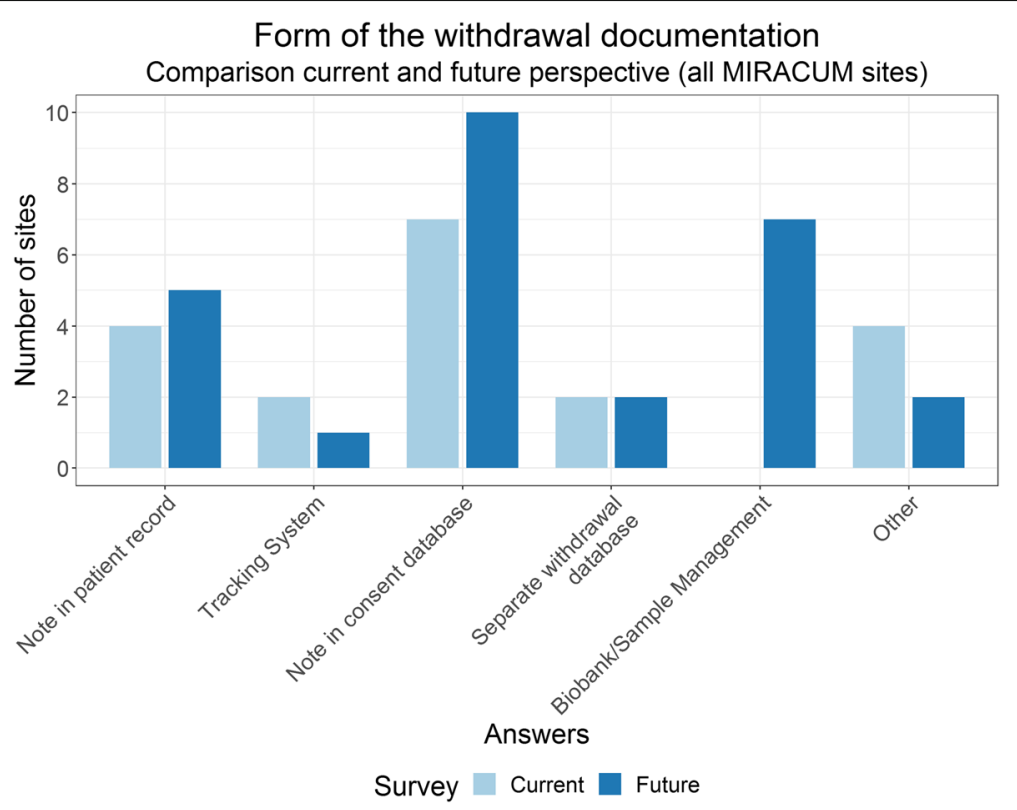

Fig. 6 Form of the withdrawal documentation. Frequency distribution of how withdrawals are documented currently and in future perspective 
Table 3 Response to the question: "Who has access to the patient consents?"

\begin{tabular}{lllllll}
\hline & Trusted third party staff & Every employee & Administrator & Study staff & Study monitors & Other \\
\hline Current & 2 & 3 & 0 & 8 & 5 & 1 \\
Future & 8 & 3 & 5 & 7 & 3 & 1
\end{tabular}

11. In response to the question, "Is it intended that a patient withdraw and at the same time already existing data continue to be available for research?" (see Table 5) Nine sites answer "yes". A suitable consent template can implement this. We support the sites in creating a suitable template to implement this case.

In summary, the analysis of the current and the future perspective of consent management at the MIRACUM sites yielded indications for the following feature requests and improvements for the consent management with gICS.

1. All sites, taking the corresponding question, aim to implement a quality assurance process for informed consents. Thus, the consent management has to be enabled to extensively support and document the process of quality assurance for consents.

2. We observed that the future documentation of the patient information will primarily be digital. However, paper-based approaches will remain relevant. Consequently, the consent management has to provide support for the documentation of the patient information process for both modalities.

3. In case of a legal review, all sites aim to store various information after successfully implementing a patient's withdrawal, such as the type of withdrawal (complete, partial), date of receipt, date of implementation, follow-up information and related pseudonyms. As a result, a focus point will be the formalised input of the information for proper documentation as part of the consent management in conformance to the "right to be forgotten" (GDPR Art. 17 [18]).

4. Today, paper-based consent processes are widespread. It is required handing over a copy of the filled in consent form to the patient (GDPR Art. 15 (3) (no specification of the form)) [19]. In the future, both, the paper-based and purely digital processes, will be equally relevant. Consequently, the consent management has to be able to generate an identical printout-version of digital consent.

5. The shift from a purely paper-based to a combination of digital and paper-based consent requires new functionalities to obtain, store, and retrieve the patient's signature. Among other technical possibilities, five out of ten sites plan to use signature pads to obtain the patient's digital signature. For this reason, comprehensive support for capturing signatures on mobile devices and other improvements is required for future consent management in the MIRACUM consortium.

6. The realisation of a partial or complete withdrawal is a complex process and requires communication efforts between various systems and organizational units. Thus, the consent management has to facilitate sending individual notifications to a configurable set of other IT-systems.

7. For legal conformity, all sites have to implement a consent withdrawal process. Thus, the management of the broad modular consent of the MII shall provide necessary guidance and shall be enabled to document partial and complete withdrawals from patients comprehensively.

8. As of today, most sites utilize various solutions to scan and digitalise consent information from paperbased consents. For a more homogenous approach, the consent management needs to automatically retrieve the patient's consent information from a proper scan (e.g. which consent modules were accepted or declined by the patient).

As a final step, we compared the current gICS-feature set $[20,21]$ with the detected relevant points resulting in the following feature requests, improvements and conceptual supports for the consent management in MIRA CUM (Table 6).

\section{Discussion}

Feature requests, improvements and conceptual support were derived from the surveys' responses with a sum of

Table 4 Response to the question: "What forms of withdrawal are used?"

\begin{tabular}{lllll}
\hline & Complete withdrawal & Partial withdrawal & Contact ban (re-contact denied) & No differentiation \\
\hline Current & 7 & 7 & 5 & 3 \\
Future & 9 & 9 & 8 & 1 \\
\hline
\end{tabular}


Table 5 Response to the question: "Is it intended that a patient withdraw and at the same time already existing data continue to be available for research?"

\begin{tabular}{lll}
\hline & Yes & No \\
\hline Current & 8 & 2 \\
Future & 9 & 1 \\
\hline
\end{tabular}

46 questions and three topics (patient information, consent retrieval and documentation, withdrawal processes) at each site. All sites had the task of filling out the corresponding questionnaires and thus presenting the site-specific actual and target status of the respective research institute, department or research project. We did not intend to compare MIRACUM sites in terms of quantities for specific projects, departments or institutes, so no restrictions were set in this respect. The majority of the questions was formulated independently from specific quantities. As a result, the answers refer to different organisational units within the partner institutions. However, the results reveal changes and tendencies based on similarities and differences between the MIRA CUM sites. Thus, we have gained insights into the current and future perspective and the necessary features and improvements of gICS to achieve the desired target state.

All centres provided feedback that shows that the work with the very detailed surveys contributed to a greater awareness of the challenges and emerging issues regarding consent management and the most relevant problems that may arise during development and implementation. It was one goal of the survey to provoke follow-up questions and discussion. The University Medicine Greifswald, and the independent trusted third party, has gathered years of experience in consent management in a large variety of study designs and settings $[13,22,23]$. Knowledge, experience, and technology transfer will support all MIRACUM sites to establish their own TTP.

\section{Conclusion}

This paper aimed to determine the most important requirements across all MIRACUM sites in a standardized survey that deals with the handling of patient information, informed consents, and withdrawals. The first goal was a detailed assessment of the current site-specific processes in consent management (I) and the sites' target status. The survey identified the site's differences and similarities from which for future usage and development in the consent management software could be derived (II). Some requirements are already implemented in gICS, but a number of new features need to be programmed for full implementation (III).

Extended and improved versions of gICS will be released continuously with several new releases per year (IV). This includes extensive documentation and a description of user interface. gICS is provided as a Docker container and can therefore be quickly updated. We intend to provide a consent management solution for the MIRACUM-related feature requests and improvements by the end of the MIRACUM funding period. In addition, feature requests and improvements defined in the context of the MII will be included.

Table 6 Resulting feature requests, improvements and conceptual supports. Features that were already available in the gICS before the survey was conducted are not included in the table

\begin{tabular}{|c|c|c|}
\hline No. & Goal & Type of enhancement \\
\hline 1 & Support and documentation of the quality assurance process of consents & Feature request \\
\hline 2 & $\begin{array}{l}\text { Documentation of the patient information procedure, independent of the } \\
\text { time of consent }\end{array}$ & Feature request \\
\hline 3 & $\begin{array}{l}\text { Support for the comprehensive documentation of successfully implemented } \\
\text { withdrawals while conforming to the "right to be forgotten" [18] }\end{array}$ & Feature request \\
\hline 4 & The improved print-out version of the digital consent for the patient & Improvement \\
\hline 5 & $\begin{array}{l}\text { Use of Tablet PCs and signature pads for mobile collection of the patients' } \\
\text { consent (including necessary digital signatures) independent of time } \\
\text { (after patient information, during or after patient admission) }\end{array}$ & Improvement \\
\hline 6 & $\begin{array}{l}\text { Configurable notifications to communicate withdrawal receipt and } \\
\text { withdrawal implementation progress to involved systems }\end{array}$ & Improvement \\
\hline 7 & $\begin{array}{l}\text { Comprehensive guidance and support for the processing and } \\
\text { implementation of withdrawals }\end{array}$ & Feature request \\
\hline 8 & $\begin{array}{l}\text { Automatic recognition of full consent information in consent scans to } \\
\text { reduce manual efforts in paper-based consent-processes }\end{array}$ & Improvement \\
\hline 9 & Support in the configuration of the roles and rights system & Conceptual support \\
\hline 10 & $\begin{array}{l}\text { Providing a common template for partial withdrawals in collaboration with } \\
\text { working group consent }\end{array}$ & Conceptual support \\
\hline 11 & See 10 & Conceptual support \\
\hline
\end{tabular}


For more interoperability and simplified usability, a subset of workflow-related functionalities (3 of 8) with relations to record linkage and pseudonymisation procedures, will technically be implemented in the context of the already existing Trusted Third Party Dispatcher [10], which is widely applied within MIRACUM.

All analysis results, the list of identified feature requests and improvements are available for all MIRACUM-sites. The milestone plan for gICS was adjusted accordingly together with the partner sites as well. The sites confirm that with these upcoming new features and improvements the software-supported processes in the area of consent management in MIRACUM can be achieved as planned.

We successfully identified essential requirements from future consent management stakeholders. The answers provided by all partner sites of the MIRACUM consortium indicate major lines in the development of consent management processes in medical research in Germany.

\section{Abbreviations}

DIC: Data integration center; EU GDPR: EU General Data Protection Regulation; IC: Informed consent; MIl: Medical informatics initiative; TTP: Trusted Third Party

\section{Acknowledgments}

We would like to thank everyone who directly or indirectly contributed to this paper.

\section{Authors' contributions}

$\mathrm{CH}, \mathrm{MBi}$, LG drafted the manuscript. $\mathrm{MBi}, \mathrm{CH}$, TB were involved in the conception and the design of the questionnaires. MBi and AV evaluated the questionnaires. $M B i, A V$ and $C H$ developed the R-based library. $A B, A M B, A P$, $B J, C B, D S, F S, H U P, M B o, M F, M R, P H, R B, T B, T L, W H$ revised the manuscript critically. The author(s) read and approved the final manuscript.

\section{Funding}

MIRACUM (Medical Informatics in Research and Care in University Medicine) is funded in context of the Medical Informatics Funding Schema by the German Federal Ministry of Education and Research (BMBF). Funding reference number: FKZ 01ZZ1801M. Open Access funding enabled and organized by Projekt DEAL.

\section{Availability of data and materials}

The $\mathrm{gICS} \mathrm{CS}^{\circledast}$ is licensed under AGPLV3 and open source. The code is available at https://github.com/mosaic-hgw/gICS. A runnable gICS as a Docker container is available in the TMF ToolPool Gesundheitsforschung at https:// www.toolpool-gesundheitsforschung.de/produkte/gICS as well as from https://ths-greifswald.de/gics.

We will provide the German version together with an English translation of the three questionnaires upon request by the readers.

\section{Declarations}

\section{Ethics approval and consent to participate}

Not applicable.

\section{Consent for publication}

Not applicable.

\section{Competing interests}

The authors declare that they have received funding for the development of gICS, but no competing interests exist.

\section{Author details}

'Institute for Community Medicine, University Medicine Greifswald, Ellernholzstr. 1-2, 17475 Greifswald, Germany. ${ }^{2}$ Trusted Third Party of the
University Medicine Greifswald, Ellernholzstr. 1-2, 17475 Greifswald, Germany. ${ }^{3}$ Institute of Medical Informatics, Justus-Liebig-University Giessen - Medicine Faculty, Rudolf-Buchheim-Str. 6, 35392 Gießen, Germany. ${ }^{4}$ Faculty of Medicine and Medical Center, Institute of Medical Biometry and Statistics, University of Freiburg, Stefan-Meier-Str. 26, 79104 Freiburg i. Br., Germany. ${ }^{5}$ Institute for Biometrics and Medical Informatics, Otto-von-Guericke University Magdeburg, Leipziger Str. 44, 39120 Magdeburg, Germany. ${ }^{6}$ Department of Urology, University Medical Center Magdeburg, Leipziger Str. 44, 39120 Magdeburg, Germany. ${ }^{7}$ Heinrich-Lanz-Center for Digital Health, University Medicine Mannheim, Theodor-Kutzer-Ufer 1-3, 68167 Mannheim, Germany. ${ }^{8}$ Unabhängige Treuhandstelle Dresden, Bereich Medizin,

Technische Universität Dresden, Fetscherstraße 74, 01307 Dresden, Germany. ${ }^{9}$ University Hospital Giessen and Marburg, Gießen, Germany. ${ }^{10}$ Medical Center for Information and Communication Technology, Universitätsklinikum Erlangen, Krankenhausstr. 12, 91054 Erlangen, Germany. ${ }^{11}$ Medical Center, University of Freiburg, Freiburg, Germany. ${ }^{12}$ Dataintegrationcentre, Philipps-Universität Marburg, Biegenstraße 10, 35037 Marburg, Germany.

${ }^{13}$ Chair of Medical Informatics, Friedrich-Alexander-Universität

Erlangen-Nürnberg, Wetterkreuz 15, 91058 Erlangen, Germany.

Received: 13 December 2020 Accepted: 23 February 2021

Published online: 08 March 2021

References

1. Prokosch HU, Acker T, Bernarding J, Binder H, Boeker M, Boerries M, et al. MIRACUM: medical informatics in research and care in university medicine. Methods Inf Med. 2018:57(S 01):e82-91.

2. Haarbrandt B, Schreiweis B, Rey S, Sax U, Scheithauer S, Rienhoff O, et al. HiGHmed - an open platform approach to enhance care and research across institutional boundaries. Methods Inf Med. 2018;57(S 01):e66-81.

3. Winter A, Staubert S, Ammon D, Aiche S, Beyan O, Bischoff V, et al. Smart Medical Information Technology for Healthcare (SMITH). Methods Inf Med. 2018;57(S 01):e92-e105.

4. Prasser F, Kohlbacher O, Mansmann U, Bauer B, Kuhn KA. Data Integration for Future Medicine (DIFUTURE). Methods Inf Med. 2018;57(S 01):e57-65.

5. Bild R, Ganslandt T, Ihrig K, Jahns R, Merzweiler A, Schreiweis B, et al. Erster Schritt: Im Rahmen der Medizininformatik-Initiative des Bundesforschungsministeriums ist eine elektronische Abbildung von Informationen aus Patienten-Einwilligungserklärungen entwickelt worden. EHealth-Com. 2019;2_3:50-3.

6. Bild R, Bialke M, Buckow K, Ganslandt T, Ihrig K, Jahns R, et al. Towards a comprehensive and interoperable representation of consent-based data usage permissions in the German medical informatics initiative. BMC Med Inform Decis Mak. 2020;20(1):103.

7. Regulation (EU) 2016/679 of the European Parliament and of the Council of 27 April 2016 on the protection of natural persons with regard to the processing of personal data and on the free movement of such data, and repealing Directive 95/46/EC (General Data Protection Regulation) 2016/ 679/EU (2016)., Art. 7 GDPR - Conditions for consent.

8. Rau H, Geidel L, Bialke M, Blumentritt A, Langanke M, Liedtke W, et al. The generic Informed Consent Service gICS ${ }^{\circledR}$ implementation and benefits of a modular consent software tool to master the challenge of electronic consent management in research. J Transl Med. 2020;18(1):287.

9. Bialke M, Bahls T, Geidel L, Rau H, Blumentritt A, Pasewald S, et al. MAGIC: once upon a time in consent management-a FHIR ${ }^{\oplus}$ tale. J Transl Med. 2018; 16(1):256

10. Bialke M, Penndorf P, Wegner T. et al. A workflow-driven approach to integrate generic software modules in a Trusted Third Party. J Transl Med. 2015:13:176.

11. MIRACUM Consortium. MIRACOLIX Tools [Available from: https://www.mira cum.org/miracolix-tools/]. Accessed 16 Apr 2020.

12. Schaar P. Privacy by design. Identity Inf Soc. 2010;3(2):267-74

13. Grabe HJ, Assel H, Bahls T, Dorr M, Endlich K, Endlich N, et al. Cohort profile: Greifswald approach to individualized medicine (GANI_MED). J Transl Med. 2014;12:144

14. Bialke M, Bahls T, Havemann C, Piegsa J, Weitmann K, Wegner T, et al. MOSAIC--A modular approach to data management in epidemiological studies. Methods Inf Med. 2015;54(4):364-71.

15. Hampf C, Bialke M, Geidel L, Blumentritt A, Bahls T, Leddig T, et al., editors. Ja, ich will. Oder? Schritte zu einer einheitlichen Lösung für die Verwaltung von Patienteneinwilligungen mithilfe des Einwilligungsmanagers gICS. 64 
Jahrestagung der Deutschen Gesellschaft für Medizinische Informatik, Biometrie und Epidemiologie eV (GMDS); 2019; Dortmund.

16. Trusted Third Party of the University Medicine Greifswald. glCS User Manual: Trusted Third Party of the University Medicine Greifswald; 2020 [Available from: http://www.ths-greifswald.de/gics/handbuch/en].

17. Unabhängige Treuhandstelle der Universitätsmedizin Greifswald. Community 2020. Available from: https://www.ths-greifswald.de/forscher/ community/. [cited 2021 10.02.2021].

18. Regulation (EU) 2016/679 of the European Parliament and of the Council of 27 April 2016 on the protection of natural persons with regard to the processing of personal data and on the free movement of such data, and repealing Directive 95/46/EC (General Data Protection Regulation) 2016/ 679/EU (2016)., Art. 17 GDPR - Right to erasure ('right to be forgotten')

19. Regulation (EU) 2016/679 of the European Parliament and of the Council of 27 April 2016 on the protection of natural persons with regard to the processing of personal data and on the free movement of such data, and repealing Directive 95/46/EC (General Data Protection Regulation) 2016/ 679/EU (2016)., Art. 15 GDPR - Right of access by the data subject.

20. Unabhängige Treuhandstelle der Universitätsmedizin Greifswald. Consent management gICS in new version 2.10 .0 available: Unabhängige Treuhandstelle der Universitätsmedizin Greifswald; 2020 [Available from: https://www.ths-greifswald.de/en/consent-management-gics-in-newversion-2-10-0-available/]

21. Unabhängige Treuhandstelle der Universitätsmedizin Greifswald. Interface GICSService 2020 [Available from: https://www.ths-greifswald.de/gics/doc]. Accessed 11 May 2020.

22. German National Cohort C. The German National Cohort: aims, study design and organization. Eur J Epidemiol. 2014;29(5):371-82.

23. Schwaneberg T, Weitmann K, Dosch A, Seyler C, Bahls T, Geidel L, et al. Data privacy management and data quality monitoring in the German Centre for Cardiovascular Research's multicentre TranslatiOnal Registry for CardiomyopatHies (DZHK-TORCH). ESC Heart Fail. 2017;4(4):440-7.

\section{Publisher's Note}

Springer Nature remains neutral with regard to jurisdictional claims in published maps and institutional affiliations.

Ready to submit your research? Choose BMC and benefit from:

- fast, convenient online submission

- thorough peer review by experienced researchers in your field

- rapid publication on acceptance

- support for research data, including large and complex data types

- gold Open Access which fosters wider collaboration and increased citations

- maximum visibility for your research: over $100 \mathrm{M}$ website views per year

At $\mathrm{BMC}$, research is always in progress.

Learn more biomedcentral.com/submissions 